\title{
Experimental Demonstration of a Self-Optimised Multi-Bit-Rate Optical Network
}

\author{
Georgios S. Zervas ${ }^{1}$, George Zarris ${ }^{1}$, Norberto Amaya-Gonzalez ${ }^{1}$, Joan Triay ${ }^{2}$, Eduard Escalona ${ }^{1}$, \\ Yixuan Qin, Cristina Cervello-Pastor ${ }^{2}$, Reza Nejabati ${ }^{1}$, Dimitra Simeonidou ${ }^{1}$ \\ (1) High-Performance Networks Group, School of CSEE, University of Essex, UK, gzerva@essex.ac.uk \\ (2) Department of Telematics Engineering, Universitat Politecnica de Catalunya (UPC), Spain
}

\begin{abstract}
This paper proposes an innovative self-managed optical network architecture for heterogenous transparent optical networks. A self-optimized dynamic network testbed that comprises multiple multi bit-rate ligthpaths on a mesh topology that utilizes a dark fiber link has been implemented and evaluated.
\end{abstract}

\section{Introduction}

The emergence of high-bandwidth applications and the recent explosive traffic growth is driving the demand for high-capacity dynamic optical networks. One of the problems in dynamic optical networks is that physical-layer impairments are also dynamic and need to be considered when establishing new lightpaths. Therefore, several impairment-aware routing algorithms have been proposed ${ }^{1}$. These algorithms are based on the premise that the quality of any lightpath can be calculated and a route with sufficient quality for the new request is established, if available. However, so far there has been no attempt to adapt existing lightpaths (LPs) to accommodate the new LP.

In contrast, for WDM transmission, several techniques have been proposed whereby the power/OSNR is balanced for all wavelengths over a link ${ }^{2}$. Such techniques, often only consider power/OSNR requirement, without assessing other linear or non-linear impairment. They also alter the configuration of existing wavelengths on a link-by-link basis without assessing the impact on the end-to-end connection(s).

In this paper we propose and experimentally demonstrate an impairment-aware selfoptimised multi-bit-rate online network that adapts the launch power in each link of individual LPs in order to ensure a specified end-to-end QoS requirement and minimise blocking due to physical-layer impairments.

\section{Self-optimized optical network concept and architecture}

Future optical networks should be easily maintainable and their capabilities should be continuously improved and upgraded by relying as little as possible on human intervention. Selfmanaged optical network is a promising paradigm towards efficient autonomic and usercontrolled management and optimization of the increasing complexity of dynamic transparent optical networks. This paper proposes a new framework and architecture introducing a layer able to deliver self-control, self-adaptation and self-optimization on online transparent optical networks. This architecture provides the ability to perceive current physical layer conditions, lightpaths power, OSNR and Q-factor on a network-wide level and then decide and act on those conditions. Such paradigm can benefit heterogeneous network environments were the type of modulation formats, bit-rates, transport formats as well as type of devices, systems and sub-systems can increase the complexity of the network control and in turn limit the physical layer performance.

The network architecture is depicted in Fig 1. It comprises a meshed or partially meshed core network with a number of users connected to it, a physical layer monitoring and reconfiguration layer as well as an optical network selfoptimization layer on top. To describe the processes and elements of the whole architecture a detailed walkthrough analysis is provided. Initially, a user dynamically requests lightpath connectivity to route data through the core network. Such requests can vary in terms of bit-rate, required quality (e.g. loss rate, delay, jitter), source and destination. After the user has initiated the request, a network requirement parser block can translate high-level user requirements (frame error rate) to data plane specific information ( $Q$-factor and/or BER). This translation can be determined by application/protocol/data profile, which can provide further information about the frame distribution of the data to be transported, together with the environmental conditions of the physical layer (type of impairments and their influence on statistical distribution of errors). After such user's requirement translation, the Lightpath (LP) Discovery (LPD) tool, which can be deployed by the use of a Path Computation Element (PCE) identifies an available path that can be used. Then the LP Semantic Description 


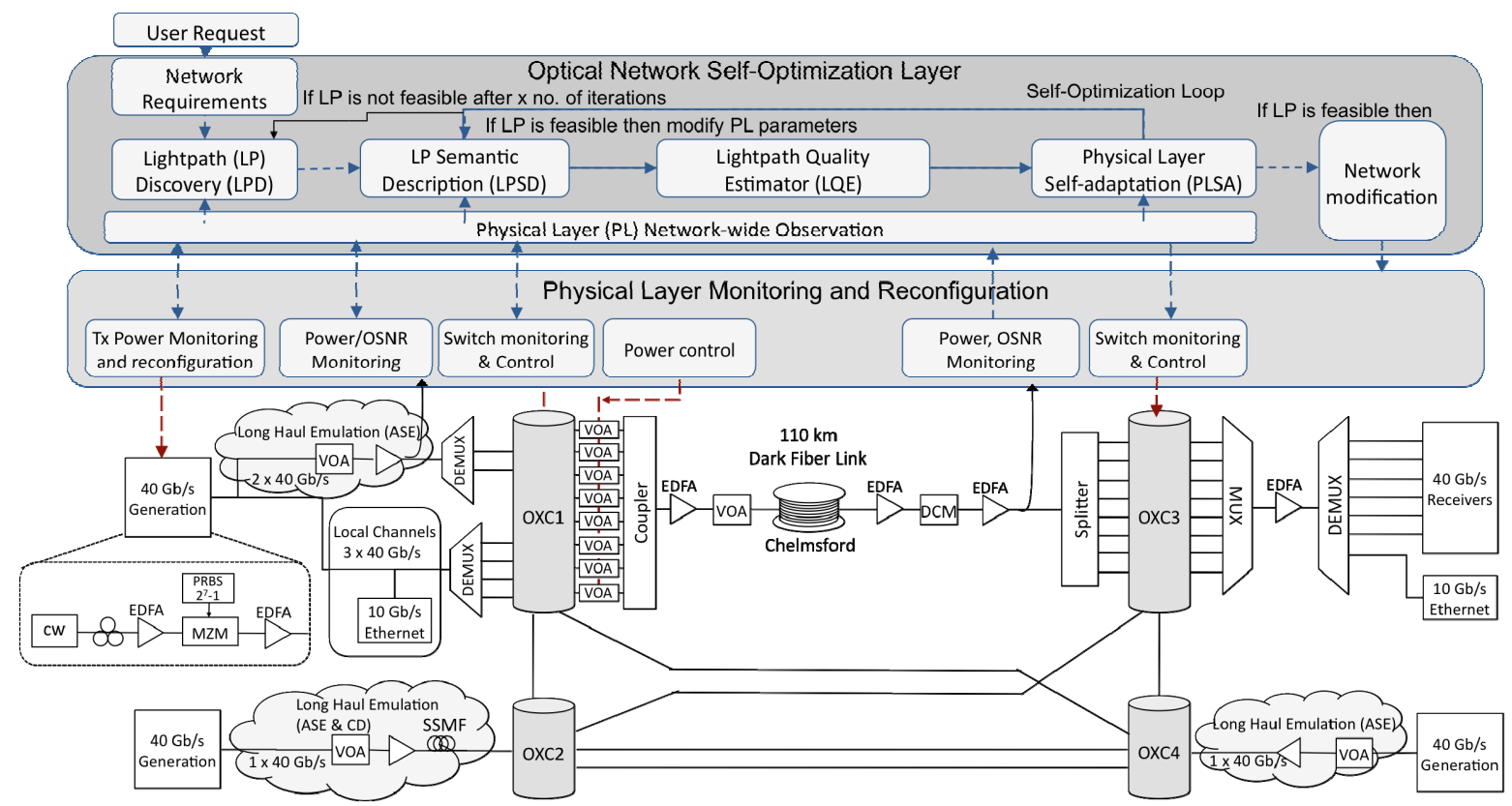

Fig. 1: Self-optimized optical network architecture and experimental setup

(LPSD) software block will provide detailed physical layer information of the candidate LP based on both predetermined values (e.g. bitrate, modulation format, etc.) but also monitored values (e.g. power across different point of the network, etc). The detailed semantic description of the LP is provided in XML format is loaded into the Ligthpath Quality Estimation (LQE) tool together with the pre-established LPs that use common links ${ }^{1}$. The LQE estimates whether or not the signal quality of the new and existing LPs (which would be affected by the addition of the new LP) would comply with the user requirements. It should be noted that the LQE computes the estimated performance of all affected LPs considering both linear and nonlinear effects that accumulate along the entire LP. Depending on estimated Q-factor values, the discovered path can either be established or not. For example, if an acceptable Q-factor value is $13.5 \mathrm{~dB}$ (assuming FEC, and allowing margin) and both the new LP and the preestablished ones are above this threshold then the LP can be reserved and configured. Otherwise the Physical layer self-adaptation (PLSA) module can evaluate all LPs' Q-factors and based on information provided from $P L$ Network-wide Observation module can identify the LP(s) that need to be optimized as well as the way to perform it (e.g. increasing power of affected LP(s) at a particular degraded link, etc.). After the theoretical alternation of such parameters, the Self-adaptation module communicates the information back (loopback) to the LPSD and in turn the LQE tool recomputes the $Q$-factor values. This process is repeated until acceptable $Q$-factor values are obtained that allow establishment of the new LP. The number of iterations should be limited to a minimum number (e.g. 2-3 times) since LQE tool is time-consuming process. However, hardwareacceleration has shown the ability to reduce its computational time ${ }^{3}$. If, on the other hand, it is determined that there are no values, that allow setting up the new lightpath with acceptable quality on requested and the existing LPs, the new connection is blocked and PCE has to calculate an alternative end-to-end path.

\section{Testbed Setup}

The setup of our proof-of-principle experiment is depicted in Fig. 1. User signals (7x40 Gb/s NRZ PRBS7 + $10 \mathrm{~Gb} / \mathrm{s}$ PRBS7) are generated and with their launched power and OSNR individually controlled are input to the meshed network through OXCs. One of the $40 \mathrm{~Gb} / \mathrm{s}$ is passed through a $2 \mathrm{Km}$ chromatic-dispersionuncompensated length of SSMF and then input to OXC2 another one is input to OXC4 and the remaining $5 \times 40 \mathrm{~Gb} / \mathrm{s}$ together with the $10 \mathrm{~Gb} / \mathrm{s}$ Ethernet are connected to OXC1. All LPs are routed through OXC1 to a 110-km dispersioncompensated dark fibre (DF) link. The launched power of each LP into the DF link is individually controlled. Last, OXC3 connects all incoming LPs to the receiver side $(40 \mathrm{~Gb} / \mathrm{s})$ or peer $(10$ $\mathrm{Gb} / \mathrm{s}$ ), where BER is measured. The OSNR of each signal was independently adjustable at the input of OXC1, and inside the DF link, by variable optical attenuator (VOA) and subsequent EDFA. In addition, the launched power of each LP into the DF link is individually 
controlled. Power and OSNR monitoring on all wavelengths is implemented at the input of OXC 1 and at the output of the DF link by utilising the WDM Measurement function of an Optical Spectrum Analyser (OSA). Measurements are taken every 2 seconds and transferred to the LPSD where they are used to automatically finetune the network model using the loopback function. When Q-factor values of all PLs are satisfactory, $L P(s)$ powers on DF link are updated and the new lightpath is established by re-configuring the optical cross-connects.

The objective of the self-optimized layer is for the LQE to estimate the launched powers of all LPs into the DF link and the objective of the selfoptimized loop to automatically adapt the launched powers on the DF link based on the monitoring information in order to enhance performance of degraded LPs and in turn have all LPs within an acceptable level $(Q>13.5)$.

\section{Experiment and results}

The experimental cases, which we are reporting were conducted as follows. First, we emulated the case in which all LPs originated from all sources are local to OXC1 sources, and therefore the OSNR of each LP at OXC1 input is similar. We then adjusted the power levels of all channels at OXC1 input in order to measure similar $Q$ (derived directly from BER measurements) for all channels. Then the preselected LQE model parameters were normalised in order to calculate the same $Q$ for one of the channels. After this, we degraded channels: $\mathrm{CH} 4, \mathrm{CH} 5, \mathrm{CH} 6$, and $\mathrm{CH} 8$ (see Fig. 2 for wavelengths) to emulate LPs with diverse signal qualities and, without altering signal powers at the OXC1 output, we measured BER on all channels from which we derived the experimental $Q$ values. The results are shown in the light coloured bars of Fig.2 (exper-case 1). The corresponding LQE values are shown in the next set of bars (LQE-case 1 ). In case 2 we selfadapted all channel power levels at the input of OXC1 and managed to equalise (to within $1 \mathrm{~dB}$ from the original $2 \mathrm{~dB}$ variation) the experimentally derived $Q$ (exper-case 2). Considering that the acceptable $Q$ is set to 13.5 $\mathrm{dB}$ in case that we don't apply self-optimization only 6 out of 8 LPs are feasible (case 1) in opposed to 8 out of 8 in case 2 (with selfoptimization). This provides a $25 \%$ improvement on blocking probability on that particular scenario. The resultant peak-to-peak power excursion was up to $6 \mathrm{~dB}$, while the total power was kept the same. For this new set of reconfigured launched power values the LQE returned the estimated $Q$ values (Fig. 2, LQE-

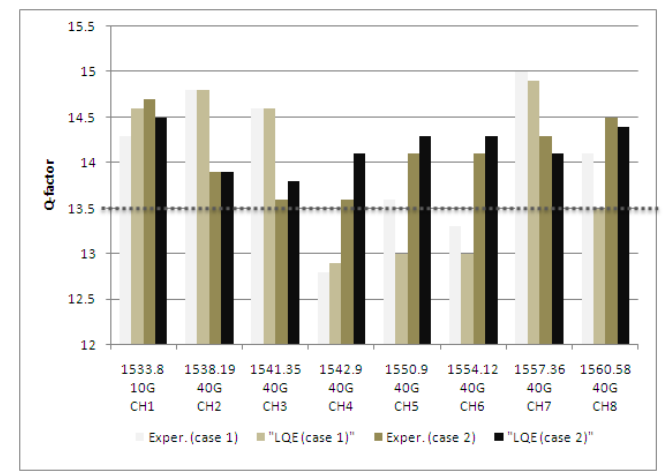

Fig. 2: Experimental vs. estimated (LQE) results without (Case1) and with (Case2) self-optimization. case2). It can be seen that the LQE-estimated $Q$ values are generally within $0.5 \mathrm{~dB}$ from those found experimentally. We observe that some of these differences appear to be systematic and may relate to the gain tilt of the EDFAs, and their noise figure dependence on wavelength, which could be taken into account in future work. In a further case (not shown in Fig. 2), we introduced dispersion on $\mathrm{CH} 8$, equivalent to 2 $\mathrm{Km}$ of standard SMF. We found experimentally that its launched power had to increase by $2 \mathrm{~dB}$ to counter the dispersion penalty; while the LQE calculated a relative power excursion of $3 \mathrm{~dB}$.

\section{Conclusions}

This paper has proposed a new self-optimized optical transport network architecture and experimentally demonstrated self-optimized network functions. The feasibility of the proposed solution has been demosntrated by delivering $0.5 \mathrm{~dB}$ accuracy between LQE and expérimental results. The potential of the architectural solution can be derived by the $1 \mathrm{~dB}$ impovement on $Q$ balancing across all channels that reduces blocking probability by $25 \%$ on the experimented case without the need to calculate and reserve alternative paths.

\section{Acknowledgements}

This work has been supported by two EU FP7 projects, GÉANT3 and DICONET.

\section{References}

$1 \mathrm{~S}$. Azodolmolky, et al. "A survey on physical layer impairments aware routing and wavelength assignment algorithms in optical networks", Computer Networks, 53(7), pp. 926-44, May. 2009.

2 A. R. Chraplyvy, J. A. Nagel, R. W. Tkach,"Equalization in amplified WDM lightwave transmission systems",IEEE PTL.,vol. 4,pp. 920 - 922, 1992

3 Y. Qin, et al. "Hardware Accelerated Impairment Aware Control Plane", OFC 2010, OWM3, Mar. 2010 